\title{
ARTICLE
}

Behavior, Psychology and Sociology

\section{Weight stigma and health behaviors: evidence from the Eating in America Study}

\author{
Kristen M. Lee ${ }^{1}$ Jeffrey M. Hunger ${ }^{2} \cdot$ A. Janet Tomiyama $\mathbb{( B )}^{1}$ \\ Received: 12 August 2020 / Revised: 18 February 2021 / Accepted: 9 April 2021 / Published online: 1 May 2021 \\ (c) The Author(s) 2021. This article is published with open access
}

\begin{abstract}
Background Weight stigma is pervasive across the U.S. and is associated with poor health outcomes including all-cause mortality. One potential reason that weight stigma may be detrimental to health is that it begets poorer health behaviors. Therefore, the present study tested for associations between weight stigma and four health behaviors (i.e., eating behavior, alcohol use, sleep disturbance, and physical activity), while controlling for BMI and other potential confounds.

Subjects/Methods Participants $(N=2022)$ in the U.S. were recruited for the Eating in America Study using a Qualtrics panel between December 2019 and January 2020 and were census-matched according to national quotas for age, gender, income, race/ethnicity, and census region. Participants completed questionnaires about weight stigma, health behaviors, demographics, and anthropometric measurements. The current study employed a two-stage investigation: exploratory analyses were first performed on a random sample of the dataset $(n=438)$, then the remaining unexamined data were used to conduct confirmatory analyses that were preregistered on the Open Science Framework.

Results Controlling for BMI, weight stigma was significantly associated with greater disordered eating $(b=0.34,95 \%$ CI $[0.31,0.38], p<0.001)$, comfort eating $(b=0.32,95 \%$ CI $[0.25,0.39], p<0.001)$, sleep disturbance $(b=0.27,95 \%$ CI $[0.20,0.33], p<0.001)$, and alcohol use $(b=0.30,95 \%$ CI [0.11, 0.49], $p=0.002)$, but not lower physical activity $(b=$ $-0.04,95 \%$ CI $[-0.13,0.05], p=0.402)$ for individuals across the weight spectrum. BMI and perceived weight status significantly moderated the effects of weight stigma on disordered eating and alcohol use. No gender differences were found. These confirmatory analyses partially replicated the exploratory stage 1 findings.

Conclusions This study provides preliminary evidence that weight stigma is linked to several poor health behaviors, which may impact physical health.
\end{abstract}

\section{Introduction}

Weight stigma is pervasive. Higher weight individuals are stigmatized across many contexts, including healthcare, employment and income, education, media, and interpersonal relationships [1]. Repeated exposure to weight stigma may be consequential as considerable evidence

Supplementary information The online version contains supplementary material available at https://doi.org/10.1038/s41366021-00814-5.

A. Janet Tomiyama

tomiyama@psych.ucla.edu

1 Department of Psychology, University of California, Los Angeles, CA, USA

2 Department of Psychology, Miami University, Oxford, OH, USA suggests that weight stigma undermines physical health even when controlling for body mass index (BMI), which addresses the potential confound that higher adiposity begets both weight stigma and poorer health. For instance, weight discrimination predicts physiological dysregulation involving a suite of outcomes (e.g., blood pressure; inflammation; cholesterol) [2], and even mortality [3]. These findings indicate a vulnerable health profile of individuals stigmatized for their weight, particularly those with overweight or obesity. Yet, it remains unclear how weight stigma may also impact several health behaviors, which is one likely pathway through which weight stigma "gets under the skin" to generate poorer health outcomes.

Scholars have previously suggested that weight stigma may play a positive role in motivating individuals to engage in health behaviors that could lead to weight loss-presumably as a way to improve health [4]. This contention, however, is not empirically supported. Rather, stigmatizing 
individuals for their weight is consistently associated with negative consequences [5]. Although health behaviors are modifiable ways to improve health across the weight spectrum, individuals who experience weight stigma may cope with this mistreatment by engaging in health compromising behaviors. For instance, a person who has been stigmatized because of their size may be less likely to go to the gym, as this is an environment that puts them at risk for further stigmatization. Individuals encountering weight stigma may also eat to comfort their distress [6]. Although previous research has shown that, independent of BMI, weight stigma is associated with increased maladaptive eating behaviors and decreased motivation to exercise [7], less is known about other common health behaviors such as alcohol use and sleep. Thus, the present study examined the relationship between weight stigma and several modifiable health behaviors in a large U.S. sample. In the next section, the existing literature on weight stigma is outlined across four different health behaviors: eating behavior, physical activity, alcohol use, and sleep. Where available, findings pertaining to gender differences in these areas of research are summarized. As will become evident below, there are notable gaps in this literature that we address in the present study.

\section{Weight stigma and eating behavior}

Eating a healthy diet helps prevent noncommunicable diseases such as diabetes, heart disease, and cancer [8]. However, weight stigma may pose one barrier to healthy eating behavior. In laboratory settings, stigmatizing individuals with higher weight (or individuals who perceived themselves as such) led to short-term increases in eating of highfat, -sugar, and -calorie foods [9]. This eating is theorized to be a form of comfort eating due to the stress of weight stigma [6] and at least one study has found an association between weight stigma and emotional eating [10].

Weight stigma is also associated with unhealthy weight control behaviors and disordered eating such as binge eating [11]. Additionally, there is some longitudinal evidence that shows the potential long-term effects of weight stigma on adolescents' eating and weight control practices. For example, one study found that adolescent girls who were labeled "too fat" showed increased bulimic and unhealthy weight control behaviors (e.g., using diet pills) 5 years later [12].

\section{Weight stigma and physical activity}

Engaging in regular physical activity is associated with numerous health benefits, including lower risk for premature all-cause mortality, hypertension, Type 2 diabetes, and cardiovascular disease [13]. For higher weight individuals, weight stigma may be a barrier to engage in physical activity, though the findings are mixed. Some studies have shown that weight stigma is associated with greater exercise avoidance [14] and lower exercise selfefficacy and intentions [15]. One study found that weight bias internalization was associated with lower levels of exercise and self-efficacy, while experienced weight stigma predicted higher levels of exercise [16]. Yet other research found a positive relationship between weight discrimination and greater sedentary behavior among middle-aged and older adults [17].

\section{Weight stigma and alcohol use}

Alcohol consumption, particularly heavy drinking, has been associated with heart disease, stroke, and some cancers [18]. There is a large body of research showing that perceived discrimination on the basis of other stigmatized identities is linked to substance use, including alcohol [19] and preliminary evidence suggests that experiencing weight stigma is associated with alcohol dependence [20]. Internalizing anti-fat attitudes may also encourage unhealthy coping mechanisms, as seen with medical students of higher weight who turn to alcohol or drugs [21]. Given the link between psychological distress and alcohol consumption [22], it is also plausible that the distress associated with weight stigma spurs unhealthy alcohol consumption.

\section{Weight stigma and sleep disturbance}

There is a noticeable lack of research in the weight stigma literature on sleep [23], but there is reason to believe a relationship may exist given research on other stigmatized identities. For example, one systematic review found strong evidence that perceived discrimination is associated with poorer sleep, from both cross-sectional and prospective data [24]. In a large, nationally representative U.S. sample, individuals who experienced more everyday discrimination had worse objective sleep and reported greater sleep difficulties [25]. Another representative study found that perceived racial discrimination in healthcare environments independently predicted sleep disturbance [26]. Individuals with higher weight may likewise experience disturbed sleep given the chronic and pervasive nature of weight stigma.

\section{Weight stigma and gender differences}

A significant portion of weight stigma research has focused on women with higher weight. The dearth of research on men is likely due in part to evidence that women are more 
likely to experience weight stigma [27]. However, recent findings indicate that weight stigma is prevalent among men as well [28]. Previous research has also shown that not all weight stigma findings related to health behaviors are uniform across genders. For example, one study found that weight teasing by family members was associated with unhealthy weight control behaviors (e.g., skipping meals, fasting) among both males and females, however only females reported extreme weight control behaviors (e.g., using laxatives, vomiting) [29]. Gender differences have also been documented in adolescence as weight-related teasing predicted greater unhealthy weight control behaviors among boys, while girls were more likely to diet [30]. In the domain of physical activity, weight stigma was associated with more vigorous physical activity among higher weight men, but not for women [31].

In sum, there is still much to be determined in the domain of weight stigma and health behaviors. The present study fills a critical gap in the extant literature by examining multiple health behaviors (i.e., physical activity, sleep, alcohol use, eating behavior) at once, across gender and weight status. It was hypothesized that: (1) Greater weight stigma would be associated with poorer health behaviors in the domains of physical activity, sleep disturbance, alcohol use, disordered eating, and comfort eating; (2) Weight status would moderate the association of weight stigma with health behaviors, such that the relationship would be stronger for individuals with higher BMIs and selfperceived weight. Given limited prior research, we made no directional hypotheses regarding gender differences. All a priori hypotheses and confirmatory analyses were preregistered on the Open Science Framework, https://osf.io/ g4wz9?view_only=d0fafe6d7cc24846b1b1cd6b59a3783e.

\section{Methods}

\section{Participants}

The UCLA North General Institutional Review Board approved the present study. Participants $(N=2022)$ in the United States were recruited for the Eating in America Study through a panel on the online survey platform, Qualtrics, between December 2019 and January 2020. Individuals who were English speaking and at least 18 years of age were eligible to participate. Individuals were census matched according to national quotas for age, self-reported gender, income, race/ethnicity, and census region in the $\mathrm{U}$. S. Eligible participants reviewed an online information sheet and gave their consent to participate.

Several steps were taken to obtain the final analytic sample in this cross-sectional study. First, a random sample $(n=500)$ was removed from the full dataset $(N=2022)$ to conduct exploratory analyses; the final exploratory sample was comprised of 438 participants after exclusion criteria were applied. Then, participants were removed from the full dataset if they met at least one of the following exclusion criteria: failed attention checks $(n=202)$, biologically implausible height $(<44$ inches or $>90$ inches; $n=25)$, weight $(<55 \mathrm{lb}$ or $>1000 \mathrm{lb} ; n=29)$, or BMI values $(<12$, or $>70 ; n=65$ ) [32]. This yielded a sample size of 1759 after removing 263 individuals. Finally, any individuals from the exploratory sample who had not already been excluded were removed to obtain the final sample $(N=1327$ participants). The exploratory sample was not included in the confirmatory analyses.

\section{Measures}

Participants completed a series of self-report measures, including assessments of daily experiences with anticipated weight stigma and weight-based discrimination, physical activity, sleep disturbance, alcohol use, disordered eating, comfort eating, demographic questions, and anthropometric measurements.

\section{Weight stigma}

Weight stigma was assessed using a 2-item composite measure of daily anticipated and experienced weight stigma (see validation work in Online Supplementary Materials 4). Individuals indicated the frequency of anticipating weight stigma ("In your day-to-day life, how often are you concerned about or worried you will be negatively stereotyped or mistreated because of your weight?") and experiencing weight stigma ("In your day-to-day life, how often are you treated with less respect, harassed, or discriminated against because of your weight?") on a 4-point response scale from (Not at all-Often). A mean score was computed $(\alpha=0.86)$.

\section{Physical activity}

Physical activity was measured with the validated [33], single item Stanford Leisure-Time Activity Categorical Item (LCAT), which comprises six activity categories ranging from sedentary to very active based on national health recommendations determined by the American College of Sports Medicine and the American Heart Association. Each category corresponds to an intensity level based on metabolic equivalents (METs). Participants selected the category that best described their physical activity over the past month.

\section{Sleep disturbance}

Sleep disturbance was assessed using the 6-item Sleep Disturbance short form from the Patient-Reported 
Outcomes Measurement Information System item bank [34]. Participants reported sleep quality over the past week on a 5-point scale (Very Poor-Very Good) and frequency of sleep disturbance in the past week on a 5-point scale (Not at all-Very Much). A mean score was calculated for each individual, with higher scores indicating greater sleep disturbance $(\alpha=0.90)$.

\section{Alcohol use}

Alcohol use was measured by the 3-item Alcohol Use Disorders Identification Test-C [35]. Participants indicated quantity of drinks on a typical day in the past year using a 6point scale (0-10 or more drinks), frequency of drinking behavior in the past 30 days on a 6-point scale (Never-Six or more times a week) and frequency of binge drinking behavior in the past year on a 5-point scale (Never-Daily or Almost Daily). All three items were summed to calculate a total alcohol use score $(\alpha=0.75)$.

\section{Disordered eating}

Disordered eating was measured using the 12-item Eating Disorder Examination Questionnaire Short (EDE-QS) [36]. Participants indicated their eating and food-related behaviors, thoughts, and feelings over the past 7 days on a 4point rating scale (0-7 days or Not at all-Markedly). Response values range from 0 to 3 , with higher scores indicating higher eating disorder symptomatology. A mean disordered eating score was calculated $(\alpha=0.89)$.

\section{Comfort eating}

Comfort eating was defined as, "When feeling a negative emotion, some people eat more food and/or eat more unhealthy food than usual-a behavior described as comfort eating." Participants indicated their frequency of comfort eating by selecting the number of days they engaged in comfort eating in the last 30 days. This item is distinct from disordered eating, as measured by the EDE-QS, which assesses food intake and compulsive exercise in relation to weight and body image concerns.

\section{Perceived weight status}

We assessed perceived weight status on a 7-point Likert scale (Very Underweight-Very Overweight) in which participants identified their perceived weight category [37].

\section{Body mass index (BMI)}

BMI was calculated from self-reported height and weight and categorized as: "Underweight," < 18.5, "Normal
Weight," 18.5-24.9, "Overweight," 25-29.9, and "Obese," $>30$.

\section{Covariates}

Covariates of age, highest level of education, gender (Woman, Man, Non-Binary/Other), race/ethnicity, and BMI were included in the analyses to test the independent effect of weight stigma on the outcomes.

\section{Analytic strategy}

\section{Exploratory analyses}

We employed a two-stage investigation to run exploratory and confirmatory analyses [38]. In stage 1, exploratory analyses were conducted on a random subset $(n=438)$ to examine associations between weight stigma and the dependent variables: physical activity, sleep disturbance, alcohol use, disordered eating, and comfort eating, controlling for age, gender, race/ethnicity, education, and BMI. Linear regression models estimated for each behavioral variable found that all health behaviors except for physical activity were significantly associated with the composite weight stigma measure. These exploratory analyses appear in Online Supplementary Materials 1.

In stage 2 , only the remaining unexamined data $(N=$ 1327) were used to conduct the confirmatory analyses. As noted in the pre-registration, physical activity was included despite it not emerging as significant in preliminary analyses given the conflicting existing literature. In our analyses, we controlled for BMI to ensure that the relationship between weight stigma and health behaviors was not a result of confounder bias, that is, to ensure that this relationship did not emerge simply because higher BMI individuals report both greater weight stigma and poorer health behaviors. It is possible that BMI is instead a collider, or a variable that is caused by both our predictor and outcome variables [39]. Controlling for a collider can artificially create an association where one may not exist. Although greater weight stigma [40] and poorer health behaviors [41] can predict higher BMI, the results from our unadjusted models (see Table 3) are consistent with the adjusted models, suggesting that controlling for BMI does not introduce collider bias. We also examined BMI as a moderator of the relationship between weight stigma and health behaviors, as previous work has shown that effects of weight stigma exposure increase as weight increases [42].

\section{Statistical analyses}

Using linear regression models, the composite weight stigma score was entered as the main predictor variable and 
the following health behaviors were entered as the dependent variables in separate models: physical activity, sleep disturbance, alcohol use, disordered eating, and comfort eating. Age, education, race/ethnicity, gender, and BMI were included as a priori covariates. All variables were normally distributed, except for comfort eating in the exploratory and confirmatory samples. The skewed distributions were corrected using natural log transformations and all analyses were performed with the natural log values for comfort eating.

For moderation analyses, PROCESS macro (model \#1) [43] was used to test the effects of BMI, perceived weight status, and gender as unique moderators on the relationship between weight stigma and all health behaviors. The same covariates were included in these models. BMI was added as a covariate in the models testing perceived weight status and gender as moderators. Given that the same pattern of results emerged for perceived weight status, these analyses appear in Online Supplementary Materials 2.

\section{Results}

The overall sample $(N=1327)$ had a mean age of 47.7 years $(S D=17.2)$ with an average BMI of $28.0(S D=7.4$; range: 12.0-68.4). Table 1 lists sample characteristics for confirmatory and exploratory samples. Those with missing data were excluded from the confirmatory analyses: BMI $(n$ $=1)$, physical activity $(n=4)$, and comfort eating $(n=2)$. Confirmatory zero-order correlations among study variables appear in Table 2 and exploratory zero-order correlations and descriptive statistics appear in Table 4. In the confirmatory sample, $42 \%$ of participants reported weight stigma. Linear regressions estimated the associations between weight stigma and the continuous outcomes of physical activity, sleep disturbance, alcohol use, disordered eating, and comfort eating (Table 3). Adjusting for covariates of age, BMI, education, gender, and race/ethnicity, weight stigma was significantly associated with all outcomes, except for physical activity. These findings replicate those of the exploratory regression analyses from stage 1 of the investigation (see Online Supplementary Materials 1). Unadjusted models showed significant associations between weight stigma and all outcomes (Table 3). Eta-squared $\left(\eta^{2}\right)$ effect size calculations indicate that weight stigma uniquely accounted for $17.5 \%$ of the variance in disordered eating; $4.0 \%$ of the variance in sleep disturbance; $3.2 \%$ of the variance in comfort eating; and $0.7 \%$ of the variance for alcohol use.

Moderation models tested whether BMI modified the association of weight stigma with outcomes. BMI significantly moderated the association of weight stigma with disordered eating $(b=-0.01, S E=0.002,95 \%$ CI $[-0.01$,
Table 1 Exploratory $(N=438)$ and confirmatory $(N=1327)$ sample characteristics.

\begin{tabular}{|c|c|c|c|c|}
\hline \multirow[t]{2}{*}{ Characteristic } & \multicolumn{2}{|c|}{ Exploratory } & \multicolumn{2}{|c|}{ Confirmatory } \\
\hline & $n$ & $(\%)$ & $n$ & $(\%)$ \\
\hline \multicolumn{5}{|l|}{ Gender } \\
\hline Women & 220 & 50.2 & 669 & 50.4 \\
\hline Men & 215 & 49.1 & 656 & 49.4 \\
\hline Non-binary/Other & 3 & 0.7 & 2 & 0.2 \\
\hline \multicolumn{5}{|l|}{ Race/Ethnicity } \\
\hline White & 292 & 66.7 & 850 & 64.1 \\
\hline Black, African American & 57 & 13.0 & 162 & 12.2 \\
\hline Native American, Eskimo, Aleut & 4 & 0.9 & 19 & 1.4 \\
\hline Hispanic, Latino/a & 52 & 11.9 & 201 & 15.1 \\
\hline Asian, Asian American & 24 & 5.5 & 72 & 5.4 \\
\hline Native Hawaiian or Pacific Islander & 1 & 0.2 & 1 & 0.1 \\
\hline Biracial/Multiracial & 7 & 1.6 & 17 & 1.3 \\
\hline Other & 1 & 0.2 & 5 & 0.4 \\
\hline \multicolumn{5}{|l|}{ Education } \\
\hline Less than high school & 11 & 2.5 & 27 & 2.0 \\
\hline High school diploma or GED & 88 & 20.1 & 264 & 19.9 \\
\hline Some college, but no degree & 106 & 24.2 & 336 & 25.3 \\
\hline Associate degree & 60 & 13.7 & 178 & 13.4 \\
\hline Bachelor's degree & 113 & 25.8 & 316 & 23.8 \\
\hline Master's degree & 48 & 11.0 & 153 & 11.5 \\
\hline Doctorate or professional degree & 12 & 2.7 & 53 & 4.0 \\
\hline \multicolumn{5}{|l|}{ Income } \\
\hline Less than $\$ 25,000$ & 77 & 17.6 & 223 & 16.8 \\
\hline$\$ 25,000-\$ 49,999$ & 102 & 23.3 & 287 & 21.6 \\
\hline$\$ 50,000-\$ 74,999$ & 84 & 19.2 & 263 & 19.8 \\
\hline$\$ 75,000-\$ 99,000$ & 60 & 13.7 & 198 & 14.9 \\
\hline$\$ 100,000-\$ 149,999$ & 64 & 14.6 & 207 & 15.6 \\
\hline$\$ 150,000-\$ 199,999$ & 31 & 7.1 & 71 & 5.4 \\
\hline Over $\$ 200,000$ & 20 & 4.6 & 78 & 5.9 \\
\hline \multicolumn{5}{|l|}{ BMI category } \\
\hline "Underweight" (<18.5) & 11 & 2.5 & 49 & 3.7 \\
\hline "Normal weight" (18.5-24.9) & 142 & 32.4 & 455 & 34.3 \\
\hline “Overweight” (25-29.9) & 147 & 33.6 & 406 & 30.6 \\
\hline “Obesity” (>30) & 138 & 31.5 & 417 & 31.4 \\
\hline \multicolumn{5}{|l|}{ Perceived weight status } \\
\hline Very underweight & 3 & 0.7 & 10 & 0.8 \\
\hline Underweight & 19 & 4.3 & 48 & 3.6 \\
\hline Slightly underweight & 28 & 6.4 & 69 & 5.2 \\
\hline About the right weight & 131 & 29.9 & 426 & 32.1 \\
\hline Slightly overweight & 155 & 35.4 & 439 & 33.1 \\
\hline Overweight & 79 & 18.0 & 264 & 19.9 \\
\hline Very overweight & 23 & 5.3 & 71 & 5.4 \\
\hline
\end{tabular}

$-0.005], t(1319)=-4.45, p<0.001$; Fig. 1) and alcohol use $(b=-0.03, S E=0.01,95 \%$ CI $[-0.05,-0.01], t$ $(1319)=-2.72, p=0.007$; Fig. 2), but not the other health behaviors (see Online Supplementary Materials 3, Table 3). Using the Johnson-Neyman technique, the relationship between weight stigma and disordered eating appeared to be weaker as BMI increased, up to a BMI of 55.5 ("severe obesity") and between weight stigma and alcohol, up to a BMI of 32.09 ("obesity"). 
Table 2 Zero-order confirmatory correlations among key variables.

\begin{tabular}{llllll}
\hline Measure & 1 & 2 & 3 & 4 & 5 \\
\hline 1. Weight stigma $M=1.76(0.86)$ & & & & \\
2. Physical activity $M=2.58(1.40)$ & $-0.085^{* *}$ & & & \\
3. Sleep disturbance $M=2.65(1.03)$ & $0.276^{* * *}$ & $-0.121^{* * *}$ & & & \\
4. Alcohol use $M=2.79(2.81)$ & $0.103^{* * *}$ & 0.040 & $0.120^{* * *}$ & & \\
5. Disordered eating $M=0.77(0.63)$ & $0.544^{* * *}$ & 0.004 & $0.328^{* * *}$ & $0.220^{* * *}$ & \\
6. Comfort eating $M=6.64(8.18)$ & $0.319^{* * *}$ & $-0.118^{* * *}$ & $0.253^{* * *}$ & $0.170^{* * *}$ & $0.414^{* * *}$ \\
\hline
\end{tabular}

Missing data excluded for physical activity $(n=4)$; comfort eating $(n=2)$. Natural log values for comfort eating were used in the analyses.

$* p<0.05 ; * * p<0.01 ; * * *<<0.001$.
Lastly, exploratory moderation analyses were conducted to test the effect of gender on the relationship between weight stigma and health behaviors. No significant interaction effects were observed (see Online Supplementary Materials 3, Table 4).

\section{Discussion}

The present study employed a two-stage research investigation to examine the relationship between weight stigma and several health behaviors in a large sample of U.S. adults. As predicted, weight stigma was significantly associated with greater disordered eating, comfort eating, alcohol use, and sleep disturbance, after controlling for covariates. No such relationship was observed for physical activity.

Targeting health behaviors (e.g., eating) to achieve weight loss is common in weight-focused health promotion policies. These policies assume that individuals can improve their health by losing weight, employing weight stigma as one strategy for motivating behavior change [5]. However, our findings indicate that weight stigma is associated with poorer health behaviors, independent of BMI. Given that physical health and weight are largely shaped by factors outside of an individual's control (i.e., genetics and social determinants like socioeconomic status) [5], it is concerning that multiple behaviors, for which individuals have some control over, may be undermined by weight stigma.

Furthermore, a lower BMI may not necessarily be protective against weight stigma. In our sample, individuals across the weight spectrum, not only those with overweight or obese BMIs, reported weight stigma. In fact, moderation analyses indicated that individuals with lower BMIs showed greater disordered eating and alcohol use in the face of weight stigma. These results emerged despite individuals with higher weight reporting greater daily weight stigma. One explanation for the observed differences in health behavioral outcomes across the weight spectrum is that infrequent health behaviors may be less likely to be enacted as coping strategies. For instance, previous research has shown that alcohol use decreases as BMI increases among females with higher weight [44]. Thus, using food, instead of alcohol, may be the more common coping strategy among individuals with higher BMIs, as previous research suggests [45]. Nonetheless, the sizes of the moderation effects were very small, with some confidence intervals functionally at zero, and thus further interpretation of the present findings should only be done with caution.

Prior research has found conflicting evidence for the relationship between weight stigma and physical activity. Some studies have found that greater weight stigma is associated with short-term increases in reported exercise behavior $[16,46]$. Others have shown that weight stigma is positively correlated with increased exercise avoidance, but has no direct link to self-reported exercise [14, 47]. The current study adds to the latter base of evidence showing no relationship between weight stigma and physical activity. One possible explanation is that participants were asked about their daily experiences with weight stigma, which may not correspond to their level of physical activity over the past month. Ecological momentary assessment methodology may provide better insight into this relationship, as demonstrated by Vartanian et al. who examined health motivations following stigmatizing events in daily life [48].

Despite emerging evidence that weight stigma is prevalent among men [28], there is a lack of research on men's health outcomes related to weight stigma. In this study, moderation by gender was not observed for any outcome. These results are consistent with previous research reporting no gender differences in poor health outcomes such as mortality and obesity due to weight stigma [3, 49]. Men may also feel pressured to meet societal body standards and thus may display the same magnitude of associations between weight stigma and health behaviors. It is recommended that the null gender findings are interpreted with caution, as more research is needed.

How might weight stigma influence an individual's health behaviors? One potential mechanism is stress. 
Table 3 Regression coefficients of weight stigma on health behaviors unadjusted and adjusted for age, gender, education, race/ethnicity, and BMI .

\begin{tabular}{|c|c|c|c|c|c|c|c|c|c|c|}
\hline & \multicolumn{5}{|c|}{ Adjusted coefficient } & \multicolumn{5}{|c|}{ Unadjusted coefficient } \\
\hline & $b$ & $S E$ & $\beta$ & $95 \%$ CI of $b$ & $p$ & $b$ & $S E$ & $\beta$ & $95 \%$ CI of $b$ & $p$ \\
\hline \multicolumn{11}{|l|}{ Sleep disturbance } \\
\hline Weight stigma & 0.27 & 0.03 & 0.22 & $0.20,0.33$ & $<0.001$ & 0.33 & 0.03 & 0.28 & $0.27,0.39$ & $<0.001$ \\
\hline Age & -0.01 & 0.002 & -0.16 & $-0.01,-0.01$ & $<0.001$ & & & & & \\
\hline Gender & -0.13 & 0.05 & -0.07 & $-0.24,-0.03$ & 0.015 & & & & & \\
\hline Race/Ethnicity & -0.001 & 0.02 & -0.001 & $-0.04,0.04$ & 0.979 & & & & & \\
\hline Education & -0.05 & 0.02 & -0.08 & $-0.09,-0.02$ & 0.002 & & & & & \\
\hline BMI & 0.001 & 0.004 & 0.01 & $-0.01,0.01$ & 0.764 & & & & & \\
\hline \multicolumn{11}{|l|}{ Alcohol use } \\
\hline Weight stigma & 0.30 & 0.10 & 0.09 & $0.11,0.49$ & 0.002 & 0.34 & 0.09 & 0.10 & $0.16,0.51$ & $<0.001$ \\
\hline Age & -0.03 & 0.01 & -0.17 & $-0.04,-0.02$ & $<0.001$ & & & & & \\
\hline Gender & 0.90 & 0.15 & 0.16 & $0.60,1.20$ & $<0.001$ & & & & & \\
\hline Race/Ethnicity & 0.02 & 0.06 & 0.01 & $-0.10,0.13$ & 0.771 & & & & & \\
\hline Education & 0.10 & 0.05 & 0.05 & $0.00,0.20$ & 0.049 & & & & & \\
\hline BMI & -0.03 & 0.01 & -0.08 & $-0.05,-0.01$ & 0.007 & & & & & \\
\hline \multicolumn{11}{|l|}{ Disordered eating } \\
\hline Weight stigma & 0.34 & 0.02 & 0.47 & $0.31,0.38$ & $<0.001$ & 0.40 & 0.02 & 0.54 & $0.37,0.43$ & $<0.001$ \\
\hline Age & -0.01 & 0.001 & -0.17 & $-0.01,-0.004$ & $<0.001$ & & & & & \\
\hline Gender & -0.14 & 0.03 & -0.11 & $-0.20,-0.08$ & $<0.001$ & & & & & \\
\hline Race/Ethnicity & -0.01 & 0.01 & -0.02 & $-0.03,0.01$ & 0.473 & & & & & \\
\hline Education & 0.03 & 0.01 & 0.07 & $0.01,0.05$ & 0.003 & & & & & \\
\hline BMI & 0.01 & 0.002 & 0.09 & $0.004,0.01$ & $<0.001$ & & & & & \\
\hline \multicolumn{11}{|l|}{ Comfort eating } \\
\hline Weight stigma & 0.32 & 0.04 & 0.25 & $0.25,0.39$ & $<0.001$ & 0.40 & 0.03 & 0.32 & $0.34,0.47$ & $<0.001$ \\
\hline Age & -0.01 & 0.002 & -0.15 & $-0.01,-0.005$ & $<0.001$ & & & & & \\
\hline Gender & 0.02 & 0.06 & 0.01 & $-0.10,0.13$ & 0.787 & & & & & \\
\hline Race/Ethnicity & -0.003 & 0.02 & -0.004 & $-0.05,0.04$ & 0.882 & & & & & \\
\hline Education & 0.05 & 0.02 & 0.07 & $0.01,0.09$ & 0.009 & & & & & \\
\hline BMI & 0.01 & 0.004 & 0.10 & $0.01,0.02$ & 0.001 & & & & & \\
\hline \multicolumn{11}{|l|}{ Physical activity } \\
\hline Weight stigma & -0.04 & 0.05 & -0.02 & $-0.13,0.05$ & 0.402 & -0.14 & 0.04 & -0.09 & $-0.23,-0.05$ & 0.002 \\
\hline Age & -0.01 & 0.003 & -0.08 & $-0.01,-0.002$ & 0.006 & & & & & \\
\hline Gender & 0.18 & 0.08 & 0.07 & $0.03,0.33$ & 0.016 & & & & & \\
\hline Race/Ethnicity & -0.11 & 0.03 & -0.11 & $-0.16,-0.05$ & $<0.001$ & & & & & \\
\hline Education & 0.10 & 0.02 & 0.10 & $0.05,0.14$ & $<0.001$ & & & & & \\
\hline BMI & -0.04 & 0.01 & -0.20 & $-0.05,-0.03$ & $<0.001$ & & & & & \\
\hline
\end{tabular}

Bold indicates statistical significance $(p<0.05)$. $S E=$ standard error; $b=$ unstandardized regression coefficient; $\beta=$ standardized regression coefficient. Natural log values for comfort eating were used in the analyses. Gender was coded as: $1=$ Woman, $2=$ Man, $3=$ Non-binary/Other. Race/ethnicity was coded as: $1=$ White, $2=$ Black/African American, $3=$ Native American/Eskimo/Aleut, $4=$ Hispanic/Latinx, $5=$ Asian/AsianAmerican, $6=$ Native Hawaiian or Pacific Islander, $7=$ Biracial/Multiracial, $8=$ Other. Education was coded as: $1=$ Less than high school, $2=$ High school diploma or equivalent, $3=$ Some college, but no degree, $4=$ Associate degree, $5=$ Bachelor's degree, $6=$ Master's degree, $7=$ Doctorate or professional degree such as JD/MD. Age and BMI were entered as continuous variables.

Previous work suggests that weight stigma is stressful $[6,50]$ and experimental lab studies manipulating weight stigma have shown that individuals with higher weight, as well as those who perceive themselves as overweight, show elevated levels of the stress hormone cortisol following exposure to a weight-stigmatizing event [51, 52].
Additionally, some research has found that individuals who experience more weight-based discrimination have higher hair cortisol levels-a finding most pronounced in those at the highest BMI [53]. Individuals who experience greater stress may engage in more unhealthy coping behaviors. Indeed, stress can drive changes in behaviors such as eating, 
Fig. 1 Mean disordered eating by weight stigma, according to BMI. Mean BMI was 28.0 $(S D=7.4)$.

Fig. 2 Mean alcohol use by weight stigma, according to BMI. Mean BMI was 28.0 $(S D=7.4)$.

Table 4 Zero-order exploratory correlations among key variables.
Disordered Eating by Severity of Weight Stigma

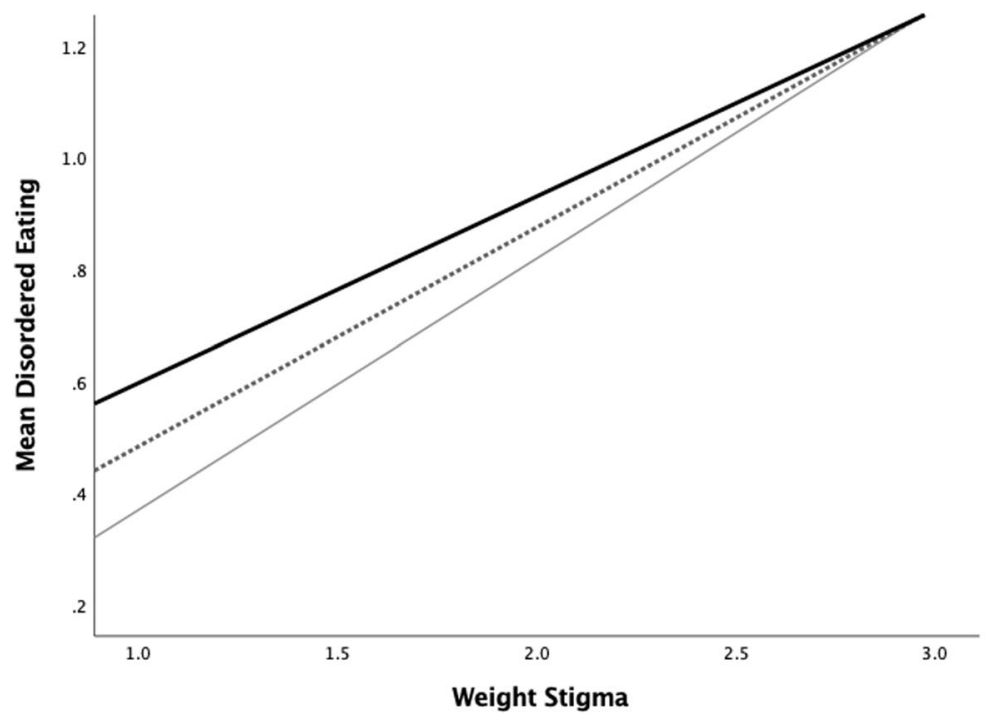

BMI

$-1 \mathrm{SD}$ $\cdots$ Mean

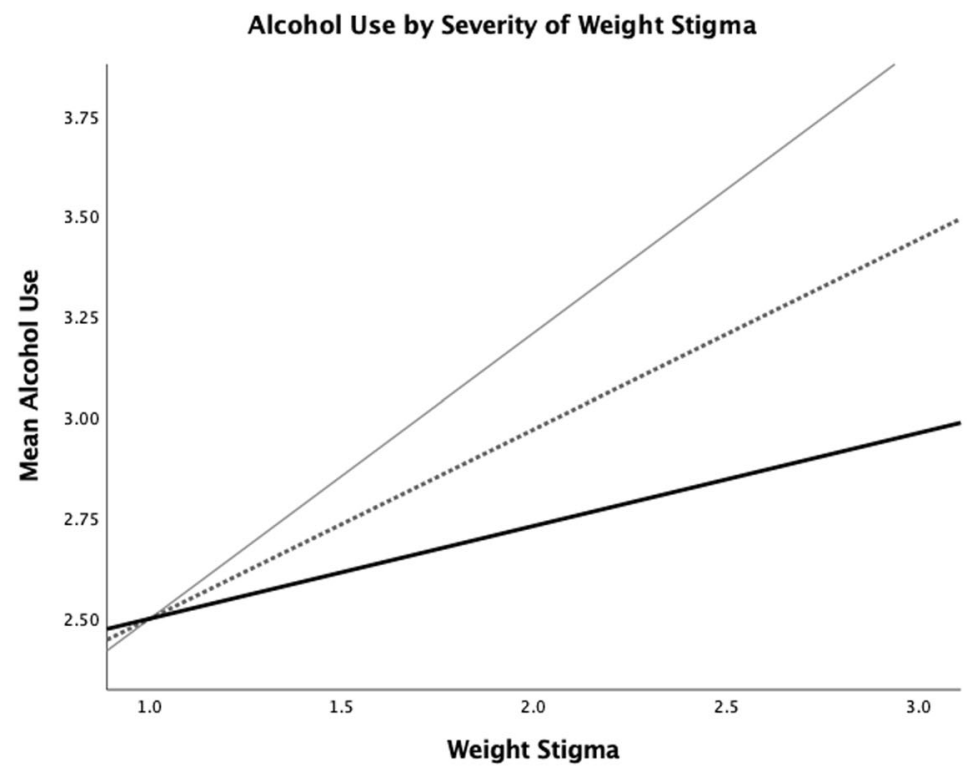

htigma

\begin{tabular}{llllll}
\hline Measure & 1 & 2 & 3 & 4 & 5 \\
\hline 1. Weight stigma $M=1.78(0.86)$ & & & & & \\
2. Physical activity $M=2.69(1.41)$ & $-0.085^{* *}$ & & & \\
3. Sleep disturbance $M=2.65(1.01)$ & $0.276^{* * *}$ & $-0.141^{* *}$ & & & \\
4. Alcohol use $M=2.76(2.71)$ & $0.153^{* *}$ & $0.097^{*}$ & $0.189^{* * * *}$ & & \\
5. Disordered eating $M=0.72(0.60)$ & $0.475^{* * *}$ & -0.066 & $0.285^{* * *}$ & $0.250^{* * *}$ & \\
6. Comfort eating $M=5.97(7.90)$ & $0.350^{* * *}$ & $-0.116^{*}$ & $0.162^{* *}$ & $0.125^{* *}$ & $0.368^{* * *}$ \\
\hline
\end{tabular}

Missing data excluded for physical activity $(n=3)$. Natural log values for comfort eating were used for the analyses.

$* p<0.05 ; * * p<0.01 ; * * * p<0.001$. 
physical activity, and sleep [54]. For example, Tataranni et al. administered synthetic cortisol vs. placebo and found greater food consumption in the cortisol group [55]. This early work is supported by accumulating evidence that cortisol is associated with increased caloric intake and greater abdominal fat storage [56]. The health behavior pathway may not be independent from that of stress but rather reflect a serial mediation model, wherein weight stigma increases stress that in turn causes decrements in health behaviors.

The present study contributes to the weight stigma literature in several ways. As noted, a key strength of this study is the assessment of several health behaviors within a large, national census-matched sample. Previous studies that have examined weight stigma in relation to different health behaviors have often had small sample sizes or were limited to female subjects. Thus, the present study may provide more generalizable information about health behaviors in the U.S. A related strength of the study is that higher BMI scores were well-represented in the sample, with $31.4 \%$ meeting BMI criteria for obesity. This is a closer estimate of the proportion of the American population that is classified with obesity (42\%) compared to previous studies [57]. Therefore, there is greater confidence that these findings reflect the experiences of individuals with obesity in the population, aiding generalizability. Lastly, we enhance reproducibility by presenting a twostage research program of exploratory and confirmatory analyses based on recommended open science practices.

There are some limitations to consider. First, a composite weight stigma score was used due to survey constraints. While early tests indicate good construct validity, additional psychometric testing is warranted (see Online Supplementary Materials 4). Another limitation is the use of selfreported weight, which is subject to inaccuracies. Additionally, the data collection period overlapped with winter holidays. Individuals may have made new year's health resolutions, and therefore the self-reported health behaviors may be more indicative of newly established goals rather than typical health habits. However, such resolutions would likely dampen, rather than magnify, the relationship between weight stigma and poor health behaviors. Lastly, the study is cross-sectional and therefore causal direction cannot be determined. Weight stigma may operate as a feedback loop that leads to weight gain through certain behaviors such as comfort eating [6], but further investigation is required. Given survey constraints, weight bias internalization was not assessed. Future research should build on these findings to determine the potential role of weight bias internalization in these health behaviors.

Despite these limitations, these study's findings show that weight stigma is significantly associated with several health behaviors. If future research confirms that this is indeed a causal relationship, weight stigma could cumulatively undermine physical health over time. Taken together, these findings highlight weight stigma as a potential barrier to healthy behaviors, and suggest that one strategy to improve population health may be to reduce weight stigma. Though more research is needed, it may be important to employ more weight-inclusive approaches to health promotion, such as removing stigmatizing language or weight outcomes from health policies and program objectives [5].

\section{Compliance with ethical standards}

Conflict of interest The authors declare no competing interests.

Publisher's note Springer Nature remains neutral with regard to jurisdictional claims in published maps and institutional affiliations.

Open Access This article is licensed under a Creative Commons Attribution 4.0 International License, which permits use, sharing, adaptation, distribution and reproduction in any medium or format, as long as you give appropriate credit to the original author(s) and the source, provide a link to the Creative Commons license, and indicate if changes were made. The images or other third party material in this article are included in the article's Creative Commons license, unless indicated otherwise in a credit line to the material. If material is not included in the article's Creative Commons license and your intended use is not permitted by statutory regulation or exceeds the permitted use, you will need to obtain permission directly from the copyright holder. To view a copy of this license, visit http://creativecommons. org/licenses/by/4.0/.

\section{References}

1. Puhl RM, Heuer CA. The stigma of obesity: a review and update. Obesity. 2009;17:941.

2. Daly M, Sutin AR, Robinson E. Perceived weight discrimination mediates the prospective association between obesity and physiological dysregulation: evidence from a population-based cohort. Psychol Sci. 2019;30:1030-9.

3. Sutin AR, Stephan Y, Terracciano A. Weight discrimination and risk of mortality. Psychol Sci. 2015;26:1803-11.

4. Callahan D. Obesity: chasing an elusive epidemic. Hastings Cent Rep. 2013;43:34-40.

5. Hunger JM, Smith JP, Tomiyama AJ. An evidence-based rationale for adopting weight-inclusive health policy. Soc Issues Policy Rev. 2020;14:73-107.

6. Tomiyama AJ. Weight stigma is stressful. A review of evidence for the Cyclic Obesity/Weight-Based Stigma model. Appetite. 2014;82:8-15.

7. Puhl RM, Himmelstein MS, Pearl RL. Weight stigma as a psychosocial contributor to obesity. Am Psychol. 2020;75:274-89.

8. Afshin A, Sur PJ, Fay KA, Cornaby L, Ferrara G, Salama JS, et al. Health effects of dietary risks in 195 countries, 1990-2017: a systematic analysis for the Global Burden of Disease Study 2017. Lancet. 2019;393:1958-72.

9. Vartanian LR, Porter AM. Weight stigma and eating behavior: a review of the literature. Appetite. 2016;102:3-14.

10. O'Brien KS, Latner JD, Puhl RM, Vartanian LR, Giles C, Griva $\mathrm{K}$, et al. The relationship between weight stigma and eating behavior is explained by weight bias internalization and psychological distress. Appetite. 2016;102:70-76. 
11. Puhl R, Suh Y. Health consequences of weight stigma: implications for obesity prevention and treatment. Curr Obes Rep. 2015;4:182-90.

12. Hunger JM, Tomiyama AJ. Weight labeling and disordered eating among adolescent girls: longitudinal evidence from the national heart, lung and blood institute growth and health study. J Adolesc Heal. 2018;63:360-2.

13. Rhodes RE, Janssen I, Bredin SS, Warburton DE, Bauman A. Physical activity: health impact, prevalence, correlates and interventions. Psychol Health. 2017;32:942-75.

14. Vartanian LR, Shaprow JG. Effects of weight stigma on exercise motivation and behavior. J Health Psychol. 2008;13:131-8.

15. Seacat JD, Mickelson KD. Stereotype threat and the exercise/ dietary health intentions of overweight women. J Health Psychol. 2009;14:556-67.

16. Pearl RL, Puhl RM, Dovidio JF. Differential effects of weight bias experiences and internalization on exercise among women with overweight and obesity. J Health Psychol. 2015;20:1626-32.

17. Jackson SE, Steptoe A. Association between perceived weight discrimination and physical activity: a population-based study among English middle-aged and older adults. BMJ Open. 2017;7: e014592.

18. Rehm J, Gmel GE Sr, Gmel G, Hasan OSM, Imtiaz S, Popova S, et al. The relationship between different dimensions of alcohol use and the burden of disease-an update. Addiction. 2017;112:968-1001.

19. Hatzenbuehler ML, Corbin WR, Fromme K. Discrimination and alcohol-related problems among college students: a prospective examination of mediating effects. Drug Alcohol Depend. 2011;115:213-20.

20. Hatzenbuehler ML, Keyes KM, Hasin DS. Associations between perceived weight discrimination and the prevalence of psychiatric disorders in the general population. Obesity. 2009;17:2033-9.

21. Phelan SM, Burgess DJ, Puhl R, Dyrbye LN, Dovidio JF, Yeazel $\mathrm{M}$, et al. The adverse effect of weight stigma on the well-being of medical students with overweight or obesity: findings from a national survey. J Gen Intern Med. 2015;30:1251-8.

22. Geisner IM, Larimer ME, Neighbors C. The relationship among alcohol use, related problems, and symptoms of psychological distress: gender as a moderator in a college sample. Addict Behav. 2004;29:843-8

23. Papadopoulos S, Brennan L. Correlates of weight stigma in adults with overweight and obesity: a systematic literature review. Obesity. 2015;23:1743-60.

24. Slopen N, Lewis TT, Williams DR. Discrimination and sleep: a systematic review. Sleep Med. 2016;18:88.

25. Owens S, Hunte H, Sterkel A, Johnson DA, Johnson-Lawrence V. Association between discrimination and objective and subjective sleep measures in the MIDUS adult sample. Psychosom Med. 2017;79:469.

26. Grandner MA, Hale L, Jackson N, Patel NP, Gooneratne NS, Troxel WM. Perceived racial discrimination as an independent predictor of sleep disturbance and daytime fatigue. Behav Sleep Med. 2012;10:235-49.

27. Puhl RM, Andreyeva T, Brownell KD. Perceptions of weight discrimination: prevalence and comparison to race and gender discrimination in America. Int J Obes. 2008;32:992-1000.

28. Himmelstein MS, Puhl RM, Quinn DM. Weight stigma in men: what, when, and by whom? Obesity. 2018;26:968-76.

29. Eisenberg ME, Berge JM, Fulkerson JA, Neumark-Sztainer D. Associations between hurtful weight-related comments by family and significant other and the development of disordered eating behaviors in young adults. J Behav Med. 2012;35:500-8.

30. Haines J, Neumark-Sztainer D, Eisenberg ME, Hannan PJ. Weight teasing and disordered eating behaviors in adolescents: longitudinal findings from Project EAT (Eating Among Teens). Pediatrics. 2006;117:e209-e215.

31. Sattler KM, Deane FP, Tapsell L, Kelly PJ. Gender differences in the relationship of weight-based stigmatisation with motivation to exercise and physical activity in overweight individuals. Heal Psychol Open. 2018;5:2055102918759691.

32. Cheng FW, Gao X, Mitchell DC, Wood C, Still CD, Rolston D, et al. Body mass index and all-cause mortality among older adults. Obesity. 2016;24:2232-9.

33. Kiernan M, Schoffman DE, Lee K, Brown SD, Fair JM, Perri MG, et al. The Stanford Leisure-Time Activity Categorical Item (LCat): a single categorical item sensitive to physical activity changes in overweight/obese women. Int $\mathrm{J}$ Obes. 2013;37:1597-602.

34. Yu L, Buysse DJ, Germain A, Moul DE, Stover A, Dodds NE, et al. Development of short forms from the PROMISTM sleep disturbance and sleep-related impairment item banks. Behav Sleep Med. 2012;10:6-24.

35. Bush K, Kivlahan DR, McDonell MB, Fihn SD, Bradley KA. The AUDIT alcohol consumption questions (AUDIT-C): an effective brief screening test for problem drinking. Arch Intern Med. 1998;158:1789-95.

36. Gideon N, Hawkes N, Mond J, Saunders R, Tchanturia K, Serpell L. Development and psychometric validation of the EDE-QS, a 12 item short form of the Eating Disorder Examination Questionnaire (EDE-Q). PLoS One. 2016;11:e0152744.

37. Robinson E, Hunger JM, Daly M. Perceived weight status and risk of weight gain across life in US and UK adults. Int $\mathrm{J}$ Obes. 2015;39:1721-6.

38. Wagenmakers EJ, Wetzels R, Borsboom D, van der Maas HL, Kievit RA. An agenda for purely confirmatory research. Perspect Psychol Sci. 2012;7:632-8.

39. Greenland S. Quantifying biases in causal models: classical confounding. Epidemiology. 2003;14:300-6.

40. Hunger JM, Tomiyama AJ. Weight labeling and obesity: a longitudinal study of girls aged 10 to 19 years. JAMA Pediatr. 2014;168:579-80.

41. Van Cauter E, Knutson KL. Sleep and the epidemic of obesity in children and adults. Eur J Endocrinol. 2008;159:59-66.

42. Hunger JM, Blodorn A, Miller CT, Major B. The psychological and physiological effects of interacting with an anti-fat peer. Body Image. 2018;27:148-55

43. Hayes AF Introduction to mediation, moderation, and conditional process analysis: a regression-based approach. The Guilford Press; 2018.

44. Kleiner KD, Gold MS, Frostpineda K, Lenzbrunsman B, Perri MG, Jacobs WS. Body mass index and alcohol use. J Addict Dis. 2004;23:105-18.

45. Puhl RM, Brownell KD. Confronting and coping with weight stigma: an investigation of overweight and obese adults*. Obesity. 2006;14:1802-15

46. Pearl RL, Dovidio JF, Puhl RM, Brownell KD. Exposure to weight-stigmatizing media: effects on exercise intentions, motivation, and behavior. J Health Commun. 2015;20:1004-13.

47. Vartanian LR, Novak SA. Internalized societal attitudes moderate the impact of weight stigma on avoidance of exercise. Obesity. 2011;19:757-62.

48. Vartanian LR, Pinkus RT, Smyth JM. Experiences of weight stigma in everyday life: Implications for health motivation. Stigma Heal. 2018;3:85.

49. Sutin AR, Terracciano A. Perceived weight discrimination and obesity. PLoS ONE. 2013;8:e70048.

50. Brewis AA. Stigma and the perpetuation of obesity. Soc Sci Med. 2014;118:152-8.

51. Incollingo Rodriguez AC, Heldreth CM, Tomiyama AJ. Putting on weight stigma: a randomized study of the effects of wearing a 
fat suit on eating, well-being, and cortisol. Obesity. 2016;24:1892-8.

52. Schvey NA, Puhl RM, Brownell KD. The stress of stigma. Psychosom Med. 2014;76:156-62.

53. Jackson SE, Kirschbaum C, Steptoe A. Perceived weight discrimination and chronic biochemical stress: a population-based study using cortisol in scalp hair. Obesity. 2016;24:2515-21.

54. Tomiyama AJ. Stress and obesity. Annu Rev Psychol. 2019;70:703-18.
55. Tataranni PA, Larson DE, Snitker S, Young JB, Flatt JP, Ravussin E. Effects of glucocorticoids on energy metabolism and food intake in humans. Am J Physiol Metab. 1996;271:E317-E325.

56. Rodriguez ACI, Epel ES, White ML, Standen EC, Seckl JR, Tomiyama AJ. Hypothalamic-pituitary-adrenal axis dysregulation and cortisol activity in obesity: a systematic review. Psychoneuroendocrinology. 2015;62:301-18.

57. Center for Disease Control and Prevention. Adult Obesity Facts. 2020. https://www.cdc.gov/obesity/data/adult.html. 